\title{
Time-Qualified Patterns of Variation of PPAR $\gamma$, DNMT1, and DNMT3B Expression in Pancreatic Cancer Cell Lines
}

\author{
Valerio Pazienza, ${ }^{1}$ Francesca Tavano, ${ }^{2}$ Massimo Francavilla, ${ }^{3}$ Andrea Fontana, ${ }^{4}$ \\ Fabio Pellegrini, ${ }^{4}$ Giorgia Benegiamo, ${ }^{1}$ Vincenzo Corbo, ${ }^{5}$ Fabio Francesco di Mola, ${ }^{2}$ \\ Pierluigi Di Sebastiano, ${ }^{2}$ Angelo Andriulli, ${ }^{1}$ and Gianluigi Mazzoccoli ${ }^{6}$
}

\author{
${ }^{1}$ Research Laboratory and Gastroenterology Unit, Scientific Institute and Regional General Hospital "Casa Sollievo della Sofferenza”, \\ 71013 San Giovanni Rotondo, Italy \\ ${ }^{2}$ Research Laboratory and Surgery Unit, Scientific Institute and Regional General Hospital "Casa Sollievo della Sofferenza”, \\ 71013 San Giovanni Rotondo, Italy \\ ${ }^{3}$ Computing Unit, Scientific Institute and Regional General Hospital "Casa Sollievo della Sofferenza", \\ 71013 San Giovanni Rotondo, Italy \\ ${ }^{4}$ Unit of Biostatistics, Scientific Institute and Regional General Hospital "Casa Sollievo della Sofferenza", \\ 71013 San Giovanni Rotondo, Italy \\ ${ }^{5}$ ARC-NET Miriam Cherubini Centre for Applied Research on Cancer, University and Hospital Trust of Verona, 37100 Verona, Italy \\ ${ }^{6}$ Division of Internal Medicine and Chronobiology Unit, Department of Medical Sciences, Scientific Institute and \\ Regional General Hospital “Casa Sollievo della Sofferenza”, 71013 San Giovanni Rotondo, Italy
}

Correspondence should be addressed to Gianluigi Mazzoccoli, g.mazzoccoli@tin.it

Received 7 June 2012; Accepted 16 July 2012

Academic Editor: Manlio Vinciguerra

Copyright () 2012 Valerio Pazienza et al. This is an open access article distributed under the Creative Commons Attribution License, which permits unrestricted use, distribution, and reproduction in any medium, provided the original work is properly cited.

\begin{abstract}
Carcinogenesis is related to the loss of homeostatic control of cellular processes regulated by transcriptional circuits and epigenetic mechanisms. Among these, the activities of peroxisome proliferator-activated receptors (PPARs) and DNA methyltransferases (DNMTs) are crucial and intertwined. PPAR $\gamma$ is a key regulator of cell fate, linking nutrient sensing to transcription processes, and its expression oscillates with circadian rhythmicity. Aim of our study was to assess the periodicity of PPAR $\gamma$ and DNMTs in pancreatic cancer (PC). We investigated the time-related patterns of PPARG, DNMT1, and DNMT3B expression monitoring their mRNA levels by qRT-PCR at different time points over a 28-hour span in BxPC-3, CFPAC-1, PANC-1, and MIAPaCa-2 PC cells after synchronization with serum shock. PPARG and DNMT1 expression in PANC-1 cells and PPARG expression in MIAPaCa-2 cells were characterized by a $24 \mathrm{~h}$ period oscillation, and a borderline significant rhythm was observed for the PPARG, DNMT1, and DNMT3B expression profiles in the other cell lines. The time-qualified profiles of gene expression showed different shapes and phase relationships in the PC cell lines examined. In conclusion, PPARG and DNMTs expression is characterized by different timequalified patterns in cell lines derived from human PC, and this heterogeneity could influence cell phenotype and human disease behaviour.
\end{abstract}

\section{Introduction}

Cancer statistics rank pancreatic cancer as the fourth leading cause of malignancy-related death worldwide [1], and incidence and mortality rates are very similar, due to difficult early diagnosis, elevated aggressiveness, and chemotherapy resistance. Bad prognosis and lack of effective treatment are responsible for high lethality, so that there is pressing need to identify molecular biomarkers for prognostic assessment and target therapy. The preservation of tissue integrity is critical for organism survival and relies on tissue renewal, driven by stem cells that are capable of responding to injury and repairing tissue damage, caused by physical, chemicals, microbial, and mutagenic agents. Transcriptional mechanisms regulate cell processes underlying cell renewal and comprising proliferation, differentiation, cell death, and 
apoptosis. Carcinogenesis relies on the loss of homeostatic mechanisms regulating cell proliferative, differentiative, and survival processes. Among the transcriptional regulators an important role is played by the peroxisome proliferatoractivated receptors (PPARs), ligand-activated transcription factors belonging to the superfamily of nuclear hormone receptors, which are considered to be involved in the regulation of nutrient metabolism and energy homeostasis, and in various pathophysiological processes, such as metabolic derangement, inflammation, and cancerogenesis [2]. PPARs are crucial for the transduction of metabolic and nutritional signals into transcriptional responses and comprise three isoforms, $\operatorname{PPAR} \alpha, \operatorname{PPAR} \beta / \delta$, and $\operatorname{PPAR} \gamma$, with a high degree of homology but with distinct biological activities [3]. PPAR $\alpha$ is mainly involved in lipid metabolism, the function of $\operatorname{PPAR} \beta / \delta$ is not entirely clear, and PPAR $\gamma$ regulates cell fate and differentiation decisions, as well as adipogenesis and fat storage [4-7]. PPAR $\gamma$ expression oscillates over a 24-hour span, and its circadian rhythmicity is crucial in the crosstalk between feeding/fasting cycles, nutrient sensing, metabolic pathways and transcriptional processes. The derangement of this crosstalk is involved in cancer development $[8,9]$. Highaffinity synthetic ligands, the thiazolidinedione, prompted the study of PPAR $\gamma$ signalling pathways in the regulation of metabolic processes and are currently evaluated as possible therapeutic tools to take advantage of $\operatorname{PPAR} \gamma$ prodifferentiative effects in cancer treatment [10].

Transcriptional processes are regulated also by epigenetic mechanisms, such as acetylation/deacetylation and methylation/demethylation. DNA methyltransferases (DNMTs) play a critical role in epigenetic mechanisms attaching methyl groups to DNA, and in particular DNMT1 keeps up the methylation pattern during DNA replication, whereas DNMT3a and DNMT3b primarily catalyze de novo methylation [11-13]. An intriguing interaction between PPAR $\gamma$ and DNMTs has been recently suggested by the downregulation of DNA methyltransferases evidenced in immune cells following ligand-dependent PPAR $\gamma$ activation [14].

The aim of our study was to assess the time-related patterns of variation of PPAR $\gamma$ and DNMTs in pancreatic cancer using in vitro models represented by pancreatic cancer cell lines evaluated after synchronization.

\section{Material and Methods}

2.1. Cell Culture and Serum Shock Procedures. BxPC-3, CFPAC-1, PANC-1, and MIA PaCa-2 cells were cultured at $37^{\circ} \mathrm{C}$ in $5 \% \mathrm{CO}_{2}$ atmosphere in DMEM medium supplemented with $10 \%$ fetal bovine serum (FCS), $100 \mathrm{U} / \mathrm{mL}$ penicillin, and $100 \mathrm{ng} / \mathrm{mL}$ streptomycin (Invitrogen Life Technologies, Milan, Italy) while CFPAC-1 and MIA PaCa2 were maintained in RPMI medium (Invitrogen Life Technologies, Milan, Italy). Cell synchronization was obtained by means of serum shock performed as follows: approximately $5 \times 10^{5}$ cells/ 6 wells were plated the day before the experiments. At the day of the experiments, culture medium was exchanged with serum-rich medium with 50\% FBS, and after 2 hours this medium was replaced as described [15]. The cells were harvested over 28 hours at the indicated time points after serum shock.

\subsection{Quantitative Real-Time Polymerase Chain Reaction.} Total RNA was extracted from BxPC-3, CFPAC-1, PANC-1, and MIA PaCa-2 cells at the indicated time points after serum shock using the RNeasy Mini Kit (Qiagen S.P.A. Milano Italy) and subsequently digested by DNase I. cDNA was synthesized from 50 ng total RNA, and quantitative Real-Time PCR (qRT-PCR) was performed using QuantiFast Sybr Green PCR kit following the one-step protocol. For real-time RTPCR, we used the following SYBR Green QuantiTect Primer purchased from Qiagen: PPARG (QT00029841), human DNMT1 (QT00034335) and DNMT3B (QT00032067). Reactions were set up in 96-well plates using a 7700 Real-Time PCR System (Applied Biosystems, Foster City, CA), and all samples were assayed in triplicate. Optical data obtained were analyzed using the default and variable parameters available in the SDS software package (version 1.9.1; Applied Biosystems, Foster City, CA). Expression levels of target gene were normalized using the housekeeping control gene TATAbinding protein (TBP, QT00000721).

2.3. Statistical Analysis. Gene expression values were normalized, for each variable for each cell line, to the expression value of the first time point (T0) of sample collection after serum shock to reduce interassay level variability. Analysis of periodicity patterns was performed, for each time series of the normalized gene expression values, by fitting a leastsquares linear regression of a single component $(24 \mathrm{~h})$ cosine waveform [16], using the MATLAB statistical package (MathWorks, Natick, Massachusetts, USA). The following parameters were estimated: "mesor" (the overall mean level of the wave); the "amplitude" ( $A$, the range from the maximum and the minimum peaks of the best-fitted curve), and the "acrophase" ( $a \varnothing$, the time in angular degrees, from the local midnight $\varnothing$, of the wave peak: acro $=$ peak). $P$-values from $F$-statistics were reported for each fitted single cosinor model, to test the null hypothesis of zero amplitude (where the wave has no periodicity). Furthermore, a novel statistical approach was employed to compare the evolution of different time qualified profiles of gene expression in the cell lines, by means of suitable statistical contrasts from a multivariate periodic linear mixed model. In particular, for each comparison, two statistical contrasts were assessed, testing whether the rhythms have an identical or opposing waveform, respectively [17]. The periodic linear mixed model can be thought as the join assessment of many different cosinor models (each one including a specific number of harmonic terms). With respect to cosinor analysis, this novel statistical approach enables the comparison of the evolution of multiple biological rhythms by jointly representing all of them in terms of sine and cosine series into a multivariate linear mixed model, taking into account all their interdependencies (intra- and interoutcome correlation structures), as well as the collection of unequally spaced measures over time and heterogeneity between gene expressions. Moreover, any specific pairwise comparison between the biological rhythms 

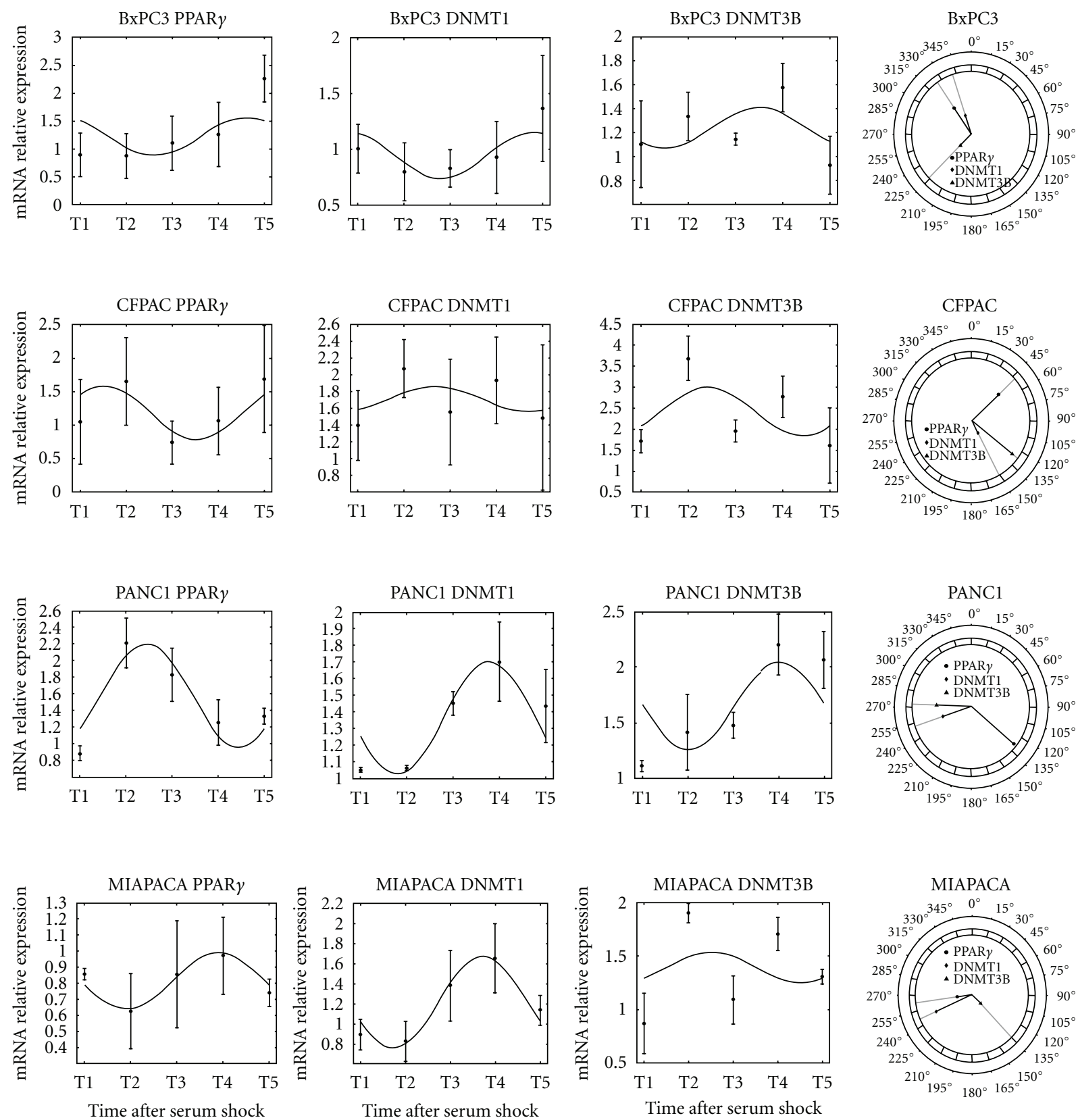

FIGURE 1: Chronograms displaying time qualified variations of PPARG, DNMT1, and DNMT3B expression level in pancreatic cancer cell lines. Original units standardized to T0 and combined for analyses. Polarograms of cosinor analysis showing the acrophases for the expression values of PPARG, DNMT1, and DNMT3B. Radial axis represents the time point (in degrees) after serum shock corresponding to the acme and vector length represents the amplitude of the oscillation.

can be performed by means of proper statistical contrasts. $P$-values $<0.05$ were considered for statistical significance. Statistical analyses were performed using MATLAB and SAS Release 9.1.3 (SAS Institute, Cary, NC).

\section{Results}

Results from cosinor analysis were reported in Table 1 and evidence a clear $24 \mathrm{~h}$ periodicity for the time-qualified variations of expression of PPARG $(P=0.016)$ and DNMT1
$(P=0.024)$ in PANC-1 cells and PPARG $(P=0.010)$ in MIA PaCa-2 cells, whereas a borderline significant rhythm was observed for the other PPARG, DNMT1, and DNMT3B expression profiles in the examined cell lines (Figure 1).

Results from multivariate periodic regression analysis were reported in Table 2. Pairwise comparisons suggested that in BxPC-3 cells the time profiles of both PPARG and DNMT1 showed flat shapes, whereas the time profiles of PPARG and DNMT3B, as well as those of DNMT1 and $D N M T 3 B$, were opposing. In CFPAC-1 cells the time profiles 
TABLE 1: Rhythm parameters from fitted single cosinor models for mRNA expression calculated on original values normalized to the first time point of sample collection after serum shock.

\begin{tabular}{|c|c|c|c|}
\hline \multicolumn{4}{|c|}{ BxPC3 } \\
\hline & PPARG & DNMT1 & DNMT3B \\
\hline Mesor & 1.22 & 0.94 & 1.24 \\
\hline Amplitude & 0.33 & 0.20 & 0.16 \\
\hline Acrophase & 325.32 & 342.87 & 225.6 \\
\hline$P$-value & 0.780 & 0.430 & 0.722 \\
\hline \multicolumn{4}{|c|}{ CFPAC } \\
\hline & PPARG & DNMT1 & DNMT3B \\
\hline Mesor & 1.18 & 1.71 & 2.42 \\
\hline Amplitude & 0.40 & 0.14 & 0.57 \\
\hline Acrophase & 47.58 & 151.69 & 127.57 \\
\hline$P$-value & 0.446 & 0.839 & 0.753 \\
\hline \multicolumn{4}{|c|}{ PANC1 } \\
\hline & PPARG & DNMT1 & DNMT3B \\
\hline Mesor & 1.58 & 1.36 & 1.65 \\
\hline Amplitude & 0.62 & 0.33 & 0.39 \\
\hline Acrophase & 129.72 & 251.25 & 272.44 \\
\hline$P$-value & 0.016 & 0.024 & 0.630 \\
\hline \multicolumn{4}{|c|}{ MIAPACA } \\
\hline & PPARG & DNMT1 & DNMT3B \\
\hline Mesor & 0.81 & 1.21 & 1.39 \\
\hline Amplitude & 0.17 & 0.45 & 0.14 \\
\hline Acrophase & 261.86 & 245.4 & 136.43 \\
\hline$P$-value & 0.010 & 0.067 & 0.933 \\
\hline
\end{tabular}

Overall gene expression levels were analyzed for time effect across the timepoints by single cosinor: fit of $24 \mathrm{~h}$ cosine to all data by least squares linear regression. Acrophase, the crest time of rhythm, is expressed in degrees. $P$-values refer to test for time effect (zero amplitude).

of all the expressions of PPARG, DNMT1, and DNMT3B were different (neither identical nor opposing). In PANC-1 cells the time profiles of PPARG and DNMT1 were different, the time profiles of PPARG and DNMT3B were opposing, and the time profiles of DNMT1 and DNMT3B had flat shapes. In MIA PaCa-2 cells the time profiles of all the expressions of PPARG, DNMT1, and DNMT3B were different (neither identical nor opposing) (Figure 2).

\section{Discussion}

Nycthemeral variations with a $24 \mathrm{~h}$ periodicity (circadian, from the Latin circa and dies) characterize behavior and physiology in the greater part of living organisms and contribute to homeostasis maintenance ensuring optimal timing of cellular phenomena in body systems, orchestrated by a complex network of transcriptional circuits [18-20]. Circadian rhythmicity is driven at the body level by a central pacemaker and master oscillator located in the hypothalamic suprachiasmatic nuclei (SCN) entrained by the light/dark cycle via the retinohypothalamic tract [21]. At the tissuespecific and single-cell levels the circadian rhythmicity is driven by molecular clocks ticked by transcription/translation feedback loops operated by a set of genes (so-called clock genes: BMAL1, CLOCK, PER 1-3, CRY 1-2) and their coded proteins, entrained by the SCN via humoral and neural outputs, and in a tissue-specific manner by other factors, such as feeding and temperature fluctuations [22-28]. The biological clocks control cell processes and tissue/organ functions driving the expression of genes coding for transcriptional factors, such as DBP (albumin D-site binding protein) and E4BP4 (adenoviral E4 protein-binding protein), which steer the expression of so-called clock controlled genes and tissue-specific output genes. The transcription factors DBP and E4BP4 among other processes control the circadian rhythmicity of PPAR $\gamma$ by binding to PPARG first exon D-sites with functional promoter activity [9].

Disruption of the circadian clock circuitry and alteration of the physiological circadian rhythmicity are considered to be involved in the processes underlying tumorigenesis [2936].

Considering the important role played in the transcriptional processes by epigenetic mechanisms such as reversible or irreversible attachment of methyl groups to DNA catalyzed by DNMTs [37] and the recently evidenced interaction between PPAR $\gamma$ and DNMTs [14], we sought to evaluate if PPAR $\gamma$ and DNMTs show correspondent oscillation in pancreatic cancer, analyzing their time-related patterns of variation in synchronized pancreatic cancer cell lines.

Our data put in evidence important differences in the periodicity and in the phase relationships of PPARG, DNMT1, and DNMT3B expression levels among the diverse 
TABLE 2: (a) Statistical contrasts from multivariate periodic regression analysis, along with a summarization of the decision to be drawn for each comparison, (b) interpretation rules: conclusions to be drawn from the joint hypotheses testing "identical biorhythms" $\left(\mathrm{H}_{01}\right)$ and "opposing biorhythms" $\left(\mathrm{H}_{02}\right)$.

(a)

\section{BxPC3 statistical contrasts}

\begin{tabular}{lcc} 
Hypotheses & $F$-value & $P$-value \\
\hline $\mathrm{H}_{01}$ : Biorhythms of PPARG and DNMT1 are identical & 0.57 & 0.695 \\
$\mathrm{H}_{02}$ : Biorhythms of PPARG and DNMT1 are opposing & 2.11 & 0.189 \\
$\mathrm{H}_{01}$ : Biorhythms of PPARG and DNMT3B are identical & 4.16 & 0.039 \\
$\mathrm{H}_{02}$ : Biorhythms of PPARG and DNMT3B are opposing & 0.46 & 0.764 \\
$\mathrm{H}_{01}$ : Biorhythms of DNMT1 and DNMT3B are identical & 11.67 & 0.001 \\
$\mathrm{H}_{02}$ : Biorhythms of DNMT1 and DNMT3B are opposing & 1.61 & 0.246 \\
\hline
\end{tabular}

Decisions derived from statistical contrasts

(1) Biorhythms of PPARG and DNMT1 have flat shapes, although statistical tests slightly suggest that they could be identical (i.e., no sufficient statistical power). (2) Biorhythms of PPARG and DNMT3B are opposing. (3) Biorhythms of DNMT1 and DNMT3B are opposing

CFPAC statistical contrasts

\begin{tabular}{lcc} 
Hypotheses & $F$-value & $P$-value \\
\hline $\mathrm{H}_{01}$ : Biorhythms of PPARG and DNMT1 are identical & 7.82 & 0.003 \\
$\mathrm{H}_{02}$ : Biorhythms of PPARG and DNMT1 are opposing & 6.70 & 0.021 \\
$\mathrm{H}_{01}$ : Biorhythms of PPARG and DNMT3B are identical & 43.78 & $<0.001$ \\
$\mathrm{H}_{02}$ : Biorhythms of PPARG and DNMT3B are opposing & 22.68 & $<0.001$ \\
$\mathrm{H}_{01}$ : Biorhythms of DNMT1 and DNMT3B are identical & 28.98 & $<0.001$ \\
$\mathrm{H}_{02}$ : Biorhythms of DNMT1 and DNMT3B are opposing & 38.02 & $<0.001$
\end{tabular}

Decisions derived from statistical contrasts

(1) Biorhythms of PPARG and DNMT1 are different (neither identical nor opposing). (2) Biorhythms of PPARG and DNMT3B are different (neither identical nor opposing). (3) Biorhythms of DNMT1 and DNMT3B are different (neither identical nor opposing)

PANC1 statistical contrasts

\begin{tabular}{lcc} 
Hypotheses & $F$-value & $P$-value \\
\hline $\mathrm{H}_{01}$ : Biorhythms of PPARG and DNMT1 are identical & 52.22 & $<0.001$ \\
$\mathrm{H}_{02}$ : Biorhythms of PPARG and DNMT1 are opposing & 5.78 & 0.010 \\
$\mathrm{H}_{01}$ : Biorhythms of PPARG and DNMT3B are identical & 16.14 & 0.002 \\
$\mathrm{H}_{02}$ : Biorhythms of PPARG and DNMT3B are opposing & 3.16 & 0.070 \\
$\mathrm{H}_{01}$ : Biorhythms of DNMT1 and DNMT3B are identical & 1.96 & 0.196 \\
$\mathrm{H}_{02}$ : Biorhythms of DNMT1 and DNMT3B are opposing & 3.57 & 0.063 \\
\hline
\end{tabular}

Decisions derived from statistical contrasts

(1) Biorhythms of PPARG and DNMT1 are different (neither identical nor opposing). (2) Biorhythms of PPARG and DNMT3B are opposing, although statistical tests slightly suggest that they could be different at all. (3) Biorhythms of DNMT1 and DNMT3B have flat shapes, although statistical tests slightly suggest that they could be identical

MIAPACA statistical contrasts

\begin{tabular}{lcc} 
Hypotheses & $F$-value & $P$-value \\
\hline $\mathrm{H}_{01}$ : Biorhythms of PPARG and DNMT1 are identical & 11.63 & $<0.001$ \\
$\mathrm{H}_{02}$ : Biorhythms of PPARG and DNMT1 are opposing & 7.23 & 0.018 \\
$\mathrm{H}_{01}$ : Biorhythms of PPARG and DNMT3B are identical & 9.89 & 0.001 \\
$\mathrm{H}_{02}$ : Biorhythms of PPARG and DNMT3B are opposing & 8.52 & 0.003 \\
$\mathrm{H}_{01}$ : Biorhythms of DNMT1 and DNMT3B are identical & 17.71 & $<0.001$ \\
$\mathrm{H}_{02}$ : Biorhythms of DNMT1 and DNMT3B are opposing & 9.06 & 0.002 \\
\hline
\end{tabular}


(a) Continued.

Decisions derived from statistical contrasts

\begin{tabular}{lc}
\hline Hypotheses & $F$-value \\
\hline (1) Biorhythms of PPARG and DNMT1 are different (neither identical nor opposing). (2) Biorhythms of PPARG and DNMT3B are \\
different (neither identical nor opposing). (3) Biorhythms of DNMT1 and DNMT3B are different (neither identical nor opposing)
\end{tabular}

(b)

$\mathrm{H}_{01}$ "identical biorhythms"

Rejected

The biorhythms are different (neither identical nor opposing)

$\mathrm{H}_{02}$ "opposing biorhythms"
Not-rejected

The biorhythms are identical

The biorhythms have flat shape

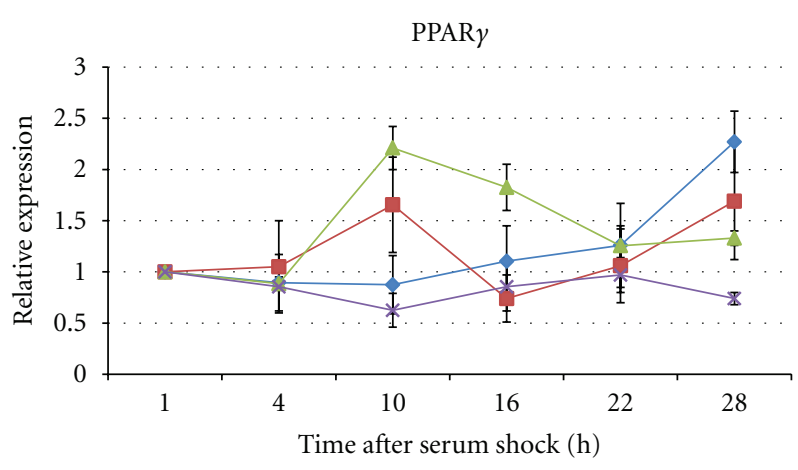
DNMT1

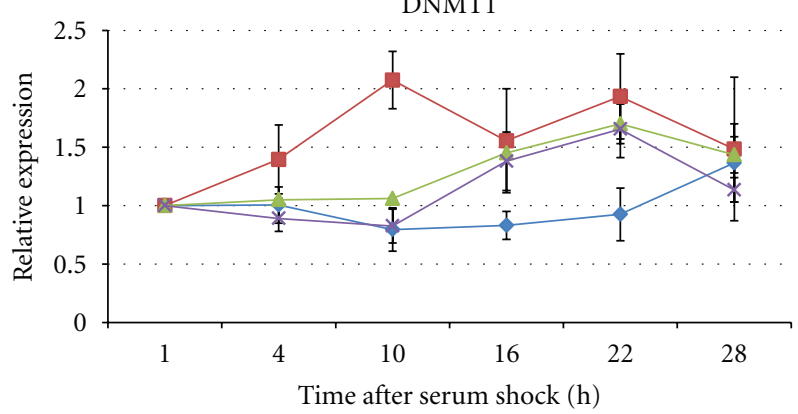
DNMT3B

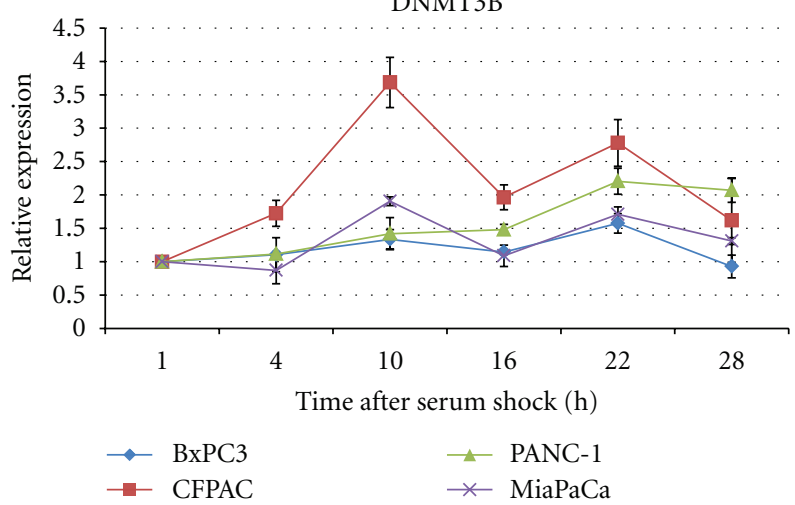

FIGURE 2: $x-y$ plots showing the time-related profiles of expression level changes of PPARG, DNMT1, and DNMT3B in pancreatic cancer cell lines. Original units standardized to T0 and combined for analyses. Gene expression data assigned to actual collection time after serum shock. cell lines examined, maybe related to a different genetic background in the diverse pancreatic cancer cells [38].

In the BxPC-3 cell line, mucin-producing cells derived from a human primary pancreatic adenocarcinoma, a borderline significant $24 \mathrm{~h}$ periodicity was evidenced for the PPARG, DNMT1, and DNMT3B expression patterns, and the time-qualified profiles of PPARG and DNMT3B, as well as the time qualified profiles of DNMT1 and DNMT3B, were opposing.

In CFPAC- 1 cells, derived from a pancreatic ductal adenocarcinoma liver metastasis of a patient with cystic fibrosis, a borderline significant rhythmicity with a $24 \mathrm{~h}$ period was found for the PPARG, DNMT1, and DNMT3B expression patterns, and the time-qualified profiles showed different shapes.

In PANC-1 cells, an epithelial-like cell line derived from a human pancreatic carcinoma, a clear $24 \mathrm{~h}$ periodicity was observed for the time qualified variations of PPARG and DNMT1 expression, a borderline significant rhythmicity with a $24 \mathrm{~h}$ period was observed for the DNMT3B expression pattern, and the time qualified profiles of $P P A R G$ and DNMT3B were opposing, whereas those of PPARG and DNMT1 were different, and the time qualified profiles of DNMT1 and DNMT3B showed flat shapes.

In the MIA PaCa-2 cell line, established from a human pancreatic adenocarcinoma, a clear 24 -h periodicity was observed for the time qualified variations of expression of $P P A R G$, and a borderline significant rhythmicity with a $24 \mathrm{~h}$ period was observed for the DNMT1 and DNMT3B expression patterns, and the time qualified profiles of $P P A R G$ and DNMT1 as well as those of PPARG and DNMT3B and the time qualified profiles of DNMT1 and DNMT3B were different (neither identical nor opposing).

The different time qualified profiles and phase relationships evidenced in the pancreatic cancer cell lines examined suggest that they rely on a dissimilar temporal architecture of transcriptional circuits and epigenetic mechanisms, which may influence cancer cell behavioral phenotype and possibly response to therapy.

Normal pancreatic duct epithelial cells do not seem to express PPAR $\gamma$, whereas human pancreatic cancer cell lines 
express the nuclear receptor, and drugs of the thiazolidinedione class transactivate the transcription of a peroxisome proliferator response element-driven promoter in a dosedependent fashion [39]. Besides, immunohistochemical staining of resected specimens by means of a polyclonal PPAR $y$ antibody has evidenced PPAR $\gamma$ protein expression in the nuclei of carcinoma cells in $90 \%$ of human pancreatic adenocarcinomas [40]. Selective PPAR $\gamma$ ligands inhibit pancreatic cancer cell growth in a dose-dependent manner and reduce the invasiveness of the tumor cells, suggesting a potential role for these agents in the adjuvant treatment of pancreatic cancer [41]. Furthermore, the first-line drug for the treatment of unresectable pancreatic cancer is represented by the nucleoside analog gemcitabine, and PPAR $\gamma$ ligands potentiate its cytotoxic action on human pancreatic cancer cells in a dosage-dependent manner and are tested to improve the prognosis of pancreatic cancer patients [42].

Inactivation of tumor suppressor genes is central to the development of all common forms of human cancer, and this inactivation often results from epigenetic silencing rather than intragenic mutations. A prevalent mechanism of tumorsuppressor gene inactivation in neoplastic disease is represented by transcriptional silencing by $\mathrm{CpG}$ island methylation, and the prototypic DNA methyltransferase, DNMT1, accounts for most methylation in mouse cells, but human cancer cells lacking DNMT1 retain significant genomic methylation and associated gene silencing [11]. In human cells, the mechanisms underlying locus-specific or global methylation patterns remain unclear, but genetic disruption of both DNMT1 and DNMT3b nearly eliminates methyltransferase activity and reduces genomic DNA methylation by greater than $95 \%$. The importance of the DNA methyltransferase DNMT1 for the maintenance of cell methylation and its role in tumorigenesis have been highlighted by genetic experiments. DNMT1 is necessary and sufficient to maintain global methylation and aberrant $\mathrm{CpG}$ island methylation in human cancer cells, and selective depletion of DNMT1 with antisense inhibitors has been shown to induce demethylation and reactivation of silenced tumor-suppressor genes such as CDKN2A. Inactivation of both DNMT1 and DNMT3B induces low levels of DNA methylation, whereas selective deletion of DNMT1 alleles in cancer cells produces clones that retain $\mathrm{CpG}$ island methylation and associated tumorsuppressor gene silencing, suggesting that the two DNMTs cooperatively maintain DNA methylation and gene silencing in human cancer cells, providing convincing support that such methylation is indispensable for best possible neoplastic proliferation $[11,35]$.

In conclusion, the cell lines derived from human pancreatic cancers are characterized by different arrays of time qualified profiles of gene expression and epigenetic modifications, which could be related to particular genetic backgrounds and could impinge on cancer cell phenotype, suggesting variable temporal organization of cell processes that could conditionate disease behaviour and response to timed delivery of conventional chemotherapy.

\section{Conflict of Interests}

The authors declare that there is no conflict of interests with respect to the authorship and/or publication of this paper.

\section{Acknowledgments}

The authors are grateful to Professor Aldo Scarpa for providing them the pancreatic cancer cell lines. The study was supported by a grant from the Italian Ministry of Health through Department of Medical Sciences, Research Laboratory and Division of Gastroenterology (RC1203GA58) and Division of Internal Medicine and Chronobiology Unit (RC1203ME46), IRCCS Scientific Institute and Regional General Hospital "Casa Sollievo della Sofferenza," Opera di Padre Pio da Pietrelcina, San Giovanni Rotondo (FG), Italy and by the " $5 \times 1000$ " voluntary contributions.

\section{References}

[1] A. Jemal, R. Siegel, E. Ward, Y. Hao, J. Xu, and M. J. Thun, "Cancer statistics, 2009," CA Cancer Journal for Clinicians, vol. 59, no. 4, pp. 225-249, 2009.

[2] P. Charoensuksai and W. Xu, "PPARs in rhythmic metabolic regulation and implications in health and disease," PPAR Research, vol. 2010, Article ID 243643, 9 pages, 2010.

[3] P. Lefebvre, G. Chinetti, J. C. Fruchart, and B. Staels, "Sorting out the roles of PPAR $\alpha$ in energy metabolism and vascular homeostasis," Journal of Clinical Investigation, vol. 116, no. 3, pp. 571-580, 2006.

[4] M. H. Hsu, U. Savas, K. J. Griffin, and E. F. Johnson, "Identification of peroxisome proliferator-responsive human genes by elevated expression of the peroxisome proliferatoractivated receptor $\alpha$ in HepG2 cells," Journal of Biological Chemistry, vol. 276, no. 30, pp. 27950-27958, 2001.

[5] J. K. Reddy, "Nonalcoholic steatosis and steatohepatitis III. Peroxisomal $\beta$-oxidation, PPAR $\alpha$, and steatohepatitis," American Journal of Physiology, vol. 281, no. 6, pp. G1333-G1339, 2001.

[6] E. Ip, G. C. Farrell, G. Robertson, P. Hall, R. Kirsch, and I. Leclercq, "Central role of PPAR $\alpha$-dependent hepatic lipid turnover in dietary steatohepatitis in mice," Hepatology, vol. 38, no. 1, pp. 123-132, 2003.

[7] S. Yu, K. Matsusue, P. Kashireddy et al., "Adipocyte-specific gene expression and adipogenic steatosis in the mouse liver due to peroxisome proliferator-activated receptor $\gamma 1$ (PPAR $\gamma 1)$ overexpression," Journal of Biological Chemistry, vol. 278, no. 1, pp. 498-505, 2003.

[8] P. Ferré, "The biology of peroxisome proliferator-activated receptors: relationship with lipid metabolism and insulin sensitivity," Diabetes, vol. 53, no. 1, supplement, pp. S43-S50, 2004.

[9] S. Takahashi, I. Inoue, Y. Nakajima et al., "A promoter in the novel exon of hPPAR $\gamma$ directs the circadian expression of PPAR $y$," Journal of Atherosclerosis and Thrombosis, vol. 17, no. 1, pp. 73-83, 2010.

[10] B. Farrow, K. L. O'Connor, K. Hashimoto, T. Iwamura, and B. M. Evers, "Selective activation of PPAR $\gamma$ inhibits pancreatic cancer invasion and decreases expression of tissue plasminogen activator," Surgery, vol. 134, no. 2, pp. 206-212, 2003. 
[11] I. Rhee, K. E. Bachman, B. H. Park et al., "DNMT1 and DNMT3b cooperate to silence genes in human cancer cells," Nature, vol. 416, no. 6880, pp. 552-556, 2002.

[12] M. Ripoli, R. Barbano, T. Balsamo et al., "Hypermethylated levels of E-cadherin promoter in Huh-7 cells expressing the HCV core protein," Virus Research, vol. 160, no. 1-2, pp. 74 81, 2011.

[13] G. Benegiamo, M. Vinciguerra, G. Mazzoccoli, A. Piepoli, A. Andriulli, and V. Pazienza, "DNA methyltransferases 1 and 3b expression in Huh-7 cells expressing HCV core protein of different genotypes," Digestive Disease Science, vol. 57, no. 6, pp. 1598-1603, 2012.

[14] J. Lei, H. Hasegawa, T. Matsumoto, and M. Yasukawa, "Peroxisome proliferator-activated receptor $\alpha$ and $\gamma$ agonists together with TGF- $\beta$ convert human CD4+CD25- T cells into functional Foxp3+ regulatory T cells," Journal of Immunology, vol. 185, no. 12, pp. 7186-7198, 2010.

[15] A. Balsalobre, F. Damiola, and U. Schibler, "A serum shock induces circadian gene expression in mammalian tissue culture cells," Cell, vol. 93, no. 6, pp. 929-937, 1998.

[16] W. Nelson, Y. L. Tong, J. K. Lee, and F. Halberg, "Methods for cosinor-rhythmometry," Chronobiologia, vol. 6, no. 4, pp. 305323, 1979.

[17] G. Mazzoccoli, A. Fontana, M. Copetti et al., "Stage dependent destructuration of neuro-endocrine-immune system components in lung cancer patients," Biomedicine and Pharmacotherapy, vol. 65, no. 1, pp. 69-76, 2011.

[18] I. Edery, "Circadian rhythms in a nutshell," Physiol Genomics, vol. 3, no. 2, pp. 59-74, 2000.

[19] T. Matsuo, S. Yamaguchi, S. Mitsui, A. Emi, F. Shimoda, and H. Okamura, "Control mechanism of the circadian clock for timing of cell division in vivo," Science, vol. 302, no. 5643, pp. 255-259, 2003.

[20] G. Mazzoccoli, V. Pazienza, and M. Vinciguerra, "Clock genes and clock controlled genes in the regulation of metabolism rhythms," Chronobiology International, vol. 29, no. 3, pp. 227251, 2012.

[21] M. H. Hastings, A. B. Reddy, and E. S. Maywood, "A clockwork web: circadian timing in brain and periphery, in health and disease," Nature Reviews Neuroscience, vol. 4, no. 8, pp. 649661, 2003.

[22] F. Damiola, N. Le Minli, N. Preitner, B. Kornmann, F. FleuryOlela, and U. Schibler, "Restricted feeding uncouples circadian oscillators in peripheral tissues from the central pacemaker in the suprachiasmatic nucleus," Genes and Development, vol. 14, no. 23, pp. 2950-2961, 2000.

[23] R. Hara, K. Wan, H. Wakamatsu et al., "Restricted feeding entrains liver clock without participation of the suprachiasmatic nucleus," Genes to Cells, vol. 6, no. 3, pp. 269-278, 2001.

[24] K. A. Stokkan, S. Yamazaki, H. Tei, Y. Sakaki, and M. Menaker, "Entrainment of the circadian clock in the liver by feeding," Science, vol. 291, no. 5503, pp. 490-493, 2001.

[25] U. Schibler and P. Sassone-Corsi, "A web of circadian pacemakers," Cell, vol. 111, no. 7, pp. 919-922, 2002.

[26] G. Mazzoccoli, “The timing clockwork of life,” Journal of Biological Regulators and Homeostatic Agents, vol. 25, no. 1, pp. 137-143, 2011.

[27] E. Filipski, V. M. King, M. C. Etienne et al., "Persistent twenty-four hour changes in liver and bone marrow despite suprachiasmatic nuclei ablation in mice," American Journal of Physiology, vol. 287, no. 4, pp. R844-R851, 2004.
[28] E. Nagoshi, C. Saini, C. Bauer, T. Laroche, F. Naef, and U. Schibler, "Circadian gene expression in individual fibroblasts: cell-autonomous and self-sustained oscillators pass time to daughter cells," Cell, vol. 119, no. 5, pp. 693-705, 2004.

[29] E. Filipski, V. M. King, X. Li et al., "Host circadian clock as a control point in tumor progression," Journal of the National Cancer Institute, vol. 94, no. 9, pp. 690-697, 2002.

[30] L. Fu and C. C. Lee, "The circadian clock: pacemaker and tumour suppressor," Nature Reviews Cancer, vol. 3, no. 5, pp. 350-361, 2003.

[31] J. J. Wille Jr., "Circadian rhythm of tumor promotion in the two-stage model of mouse tumorigenesis," Cancer Letters, vol. 190, no. 2, pp. 143-149, 2003.

[32] G. Mazzoccoli, A. Panza, M. R. Valvano et al., "Clock gene expression levels and relationship with clinical and pathological features in colorectal cancer patients," Chronobiology International, vol. 28, no. 10, pp. 841-851, 2011.

[33] G. Mazzoccoli, V. Pazienza, A. Panza et al., "ARNTL2 and SERPINE1: potential biomarkers for tumor aggressiveness in colorectal cancer," Journal of Cancer Research and Clinical Oncology, vol. 138, no. 3, pp. 501-511, 2012.

[34] G. Mazzoccoli, A. Piepoli, M. Carella et al., "Altered expression of the clock gene machinery in kidney cancer," Biomedicine \& Pharmacotherapy, vol. 66, pp. 175-179, 2012.

[35] G. Mazzoccoli, R. B. Sothern, G. Greco et al., "Time-related dynamics of variation in core clock gene expression levels in tissues relevant to the immune system," International Journal of Immunopathology and Pharmacology, vol. 24, no. 4, pp. 869879, 2011.

[36] V. Pazienza, A. Piepoli, and A. Panza, "SIRT1 and the clock gene machinery in colorectal cancer," Cancer Investigation, vol. 30, no. 2, pp. 98-105, 2012.

[37] M. F. Robert, S. Morin, N. Beaulieu et al., "DNMT1 is required to maintain $\mathrm{CpG}$ methylation and aberrant gene silencing in human cancer cells," Nature Genetics, vol. 33, no. 1, pp. 61-65, 2003.

[38] E. L. Deer, J. González-Hernández, J. D. Coursen et al., "Phenotype and genotype of pancreatic cancer cell lines," Pancreas, vol. 39, no. 4, pp. 425-435, 2010.

[39] W. Motomura, T. Okumura, N. Takahashi, T. Obara, and Y. Kohgo, "Activation of peroxisome proliferator-activated receptor $\gamma$ by troglitazone inhibits cell growth through the increase of p27(Kip1) in human pancreatic carcinoma cells," Cancer Research, vol. 60, no. 19, pp. 5558-5564, 2000.

[40] A. Elnemr, T. Ohta, K. Iwata et al., "PPARgamma ligand (thiazolidinedione) induces growth arrest and differentiation markers of human pancreatic cancer cells," International Journal of Oncology, vol. 17, no. 6, pp. 1157-1164, 2000.

[41] K. Hashimoto, R. T. Ethridge, and B. M. Evers, "Peroxisome proliferator-activated receptor $\gamma$ ligand inhibits cell growth and invasion of human pancreatic cancer cells," International Journal of Gastrointestinal Cancer, vol. 32, no. 1, pp. 7-22, 2002.

[42] H. Koga, K. Selvendiran, R. Sivakumar et al., "PPAR $\gamma$ potentiates anticancer effects of gemcitabine on human pancreatic cancer cells," International Journal of Oncology, vol. 40, no. 3, pp. 679-685, 2012. 


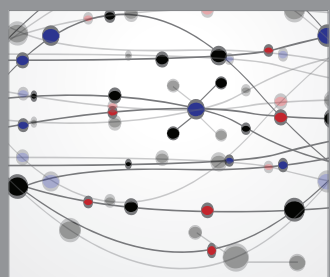

The Scientific World Journal
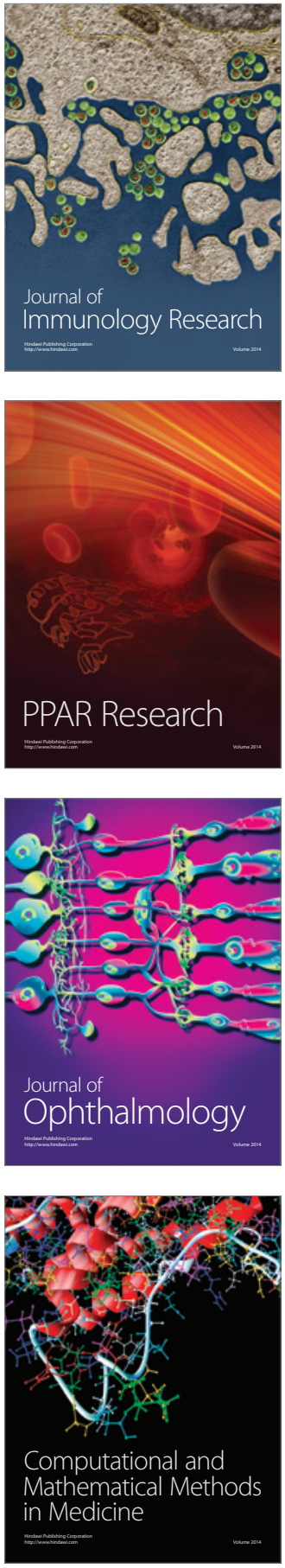

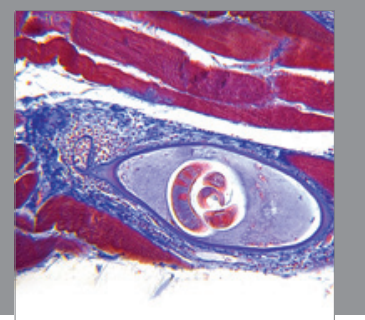

Gastroenterology

Research and Practice
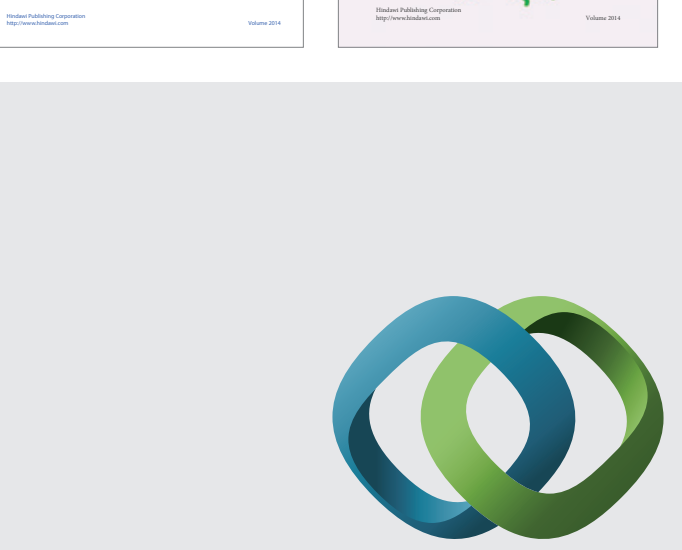

\section{Hindawi}

Submit your manuscripts at

http://www.hindawi.com
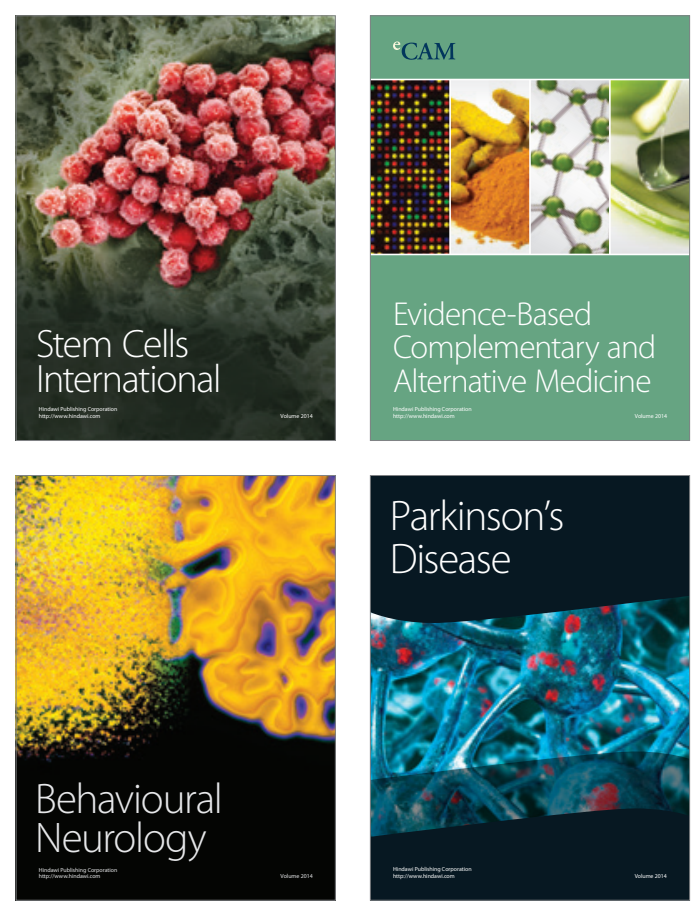

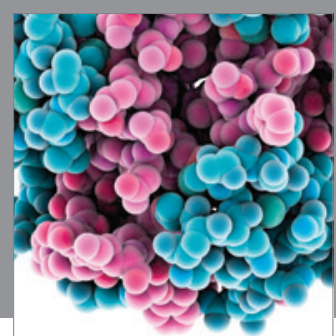

Journal of
Diabetes Research

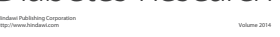

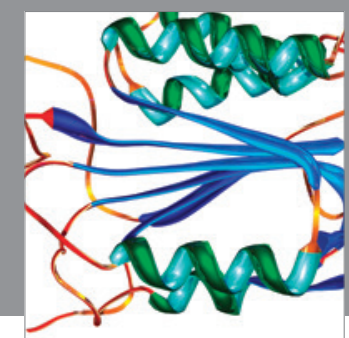

Disease Markers
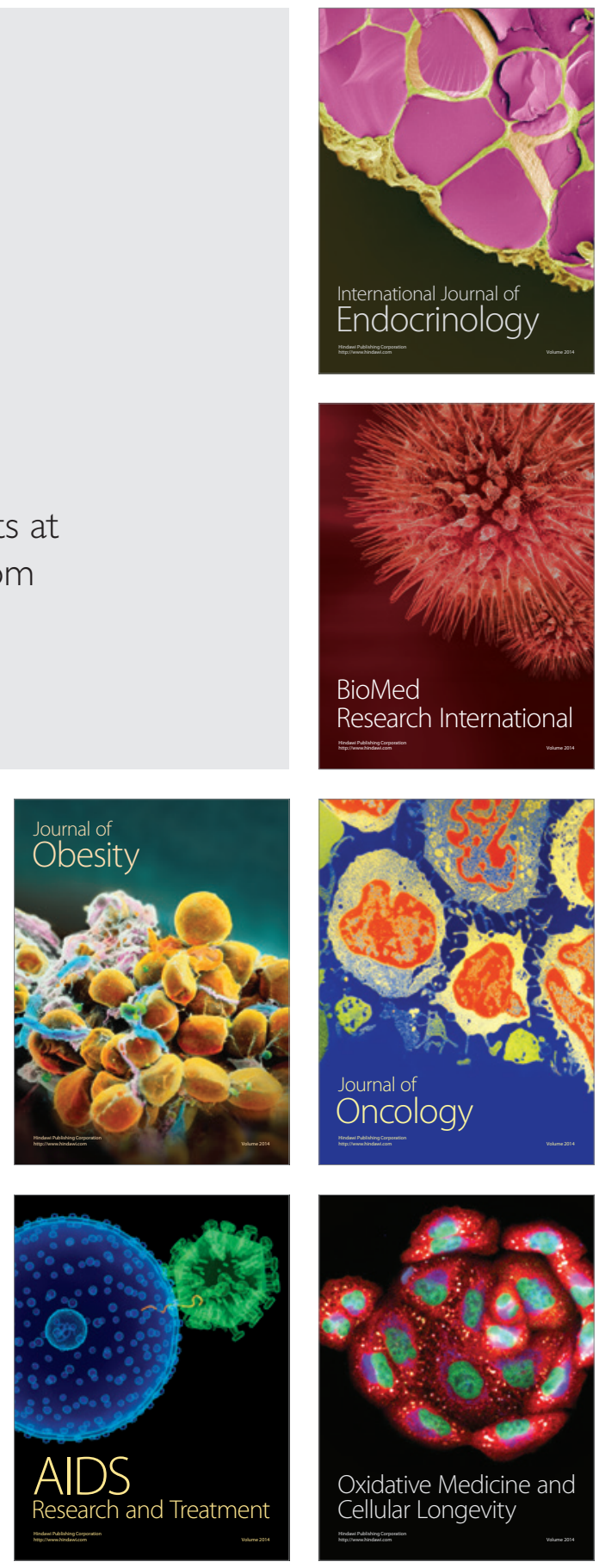